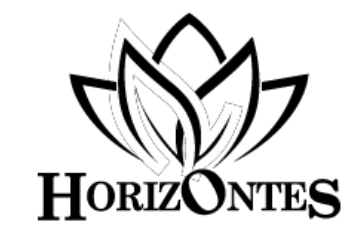

Horizontes. Revista de Investigación en Ciencias de la Educación Https: ://doi.org/10.33996/revistahorizontes.v2i5.42 enero-marzo, 2018 Volumen 2 / No. 5 pp. 22-37

www.revistahorizontes.org

\title{
Cambios en la enseñanza de los proyectos a partir de un plan de formación pedagógica
}

\section{Changes in the teaching of projects based on a pedagogical training plan}

\author{
Lisbeth Brito \\ Lisbethare06@gmail.com \\ Universidad Rafael María Baralt, Venezuela
}

\section{RESUMEN}

El presente estudio, tuvo como objetivo detectar los cambios que emergen en los docentes durante la implementación de un plan de formación pedagógica para la enseñanza de los proyectos desde la integralidad del ser humano. Se trató de una investigación de tipo interactiva bajo la comprensión holística de la ciencia, específicamente dentro de la modalidad de investigación acción, orientada a transformar la realidad de una institución que estaba en los primeros pasos del trabajo por proyecto. El diseño de la investigación fue de campo, transeccional, contemporáneo, unieventual. La técnica de recolección de los datos fue la observación participativa. Los resultados evidenciaron cambios en el contexto tales como: Manejo adecuado, durante las clases de los proyectos pedagógicos, de algunos aspectos didácticos como: estrategias, métodos $\mathrm{y}$ técnicas, acordes a las temáticas desarrolladas; además se observó la frecuencia de la integración de las dimensiones del ser humano, en la praxis de la enseñanza de los proyectos; pero aunado a ello emergió la participación activa del docente, con entusiasmo, en las diversas actividades de intercambio y socialización.

Palabras clave: Enseñanza por proyectos; formación pedagógica; integralidad del ser humano

\section{ABSTRACT}

The objective of this study was to detect the changes that emerge in teachers during the implementation of a pedagogical training plan for the teaching of projects from the integrality of the human being. It was an interactive type of research under the holistic understanding of science, specifically within the modality of action research, aimed at transforming the reality of an institution that was in the first steps of work by project. The design of the research was of field, transectional, contemporary, unieventual. The technique of data collection was participatory observation. The results showed changes in the context such as: Appropriate management, during the classes of the pedagogical projects, of some didactic aspects such as: strategies, methods and techniques, according to the developed themes; In addition, we observed the frequency of the integration of the dimensions of the human being, in the practice of teaching the projects; but coupled with this emerged the active participation of the teacher, with enthusiasm, in the various activities of exchange and socialization.

Key words: Integrality of the human being; pedagogical training; teaching by projects 


\section{INTRODUCCIÓN}

La educación, es la base fundamental de la formación académica y cultural del ser humano, a través de ella, el hombre llega a humanizarse y a cambiar su futuro al mejorar su calidad de vida; por tal razón, el docente actual tiene una enorme responsabilidad, ya que además de ser maestro tiene que ser un individuo polifacético, capaz de realizar diversas actividades que forman parte de su praxis educativa con prioridad en la investigación socioeducativa, ya que esto le permite ir en la búsqueda de respuestas para un sinnúmero de situaciones o problemas que se generan constantemente en los contextos educativos; de allí, la necesidad de incitar a la participación de la comunidad afectada o comprometida, lo cual implica la incorporación de los proyectos en la educación

De esta manera, se presentan en este trabajo los cambios que se percibieron en un grupo de docentes de una institución técnica, a partir de un programa de formación pedagógica dirigido a atender las necesidades de los docentes luego de la transformación curricular que se implementó desde el inicio del sistema educativo bolivariano en Venezuela.

La sociedad moderna, en su expectativa por el proceso de globalización, cada día se hace más exigente en cuanto a las funciones y el desarrollo social del ciudadano; lo cual provoca que en la educación se desarrollen nuevas maneras de aprender, por ser indudablemente la vía más directa para promocionar los diferentes cambios que surgen en el mundo orientados a seguir en el proceso de evolución social.

En este sentido Peiró (2005), plantea a la educación como un proceso global y continuo donde los contenidos educativos están ligados a la forma como se relacionan las instituciones educativas con la sociedad, la comunidad y los aspectos que se pueden transformar; es decir, puede concebirse como un instrumento de cambio social con vista al logro de una mayor justicia en el contexto

Por otra parte, Felfeto (2014), expone que la educación es un "proceso entre diversos elementos que implica muchos más que transmitir conocimientos y que normalmente se lleva a cabo en un individuo a lo largo de la vida" (p.45). En virtud de ello, se puede afirmar que educar a un individuo es un proceso del continuo humano; que implica estar en consonancia con los hechos que se vislumbran en el momento para el cual se esté llevando el proceso, tomando en consideración la importancia del hombre de satisfacer sus necesidades con la finalidad de alcanzar la calidad de vida dentro de su entorno.

En relación a esto, diferentes países de América del Sur, tales como Bolivia, Argentina y Ecuador en los últimos años permanecen en constante transformación de sus sistemas educativos, con el objetivo de lograr sincronizarse con las filosofías emergentes, producto del continuo social que a su vez está guiado por las demandas de la actual sociedad del conocimiento; en la búsqueda de avanzar y posicionarse como países en vías de desarrollo.

Por su parte, Venezuela en las últimas décadas ha realizado grandes cambios en su sistema educativo, lo cual ha obligado, a las diferentes instituciones educativas públicas del país, a desarrollar estrategias orientadas a cumplir con los requerimientos y normativas que el ente rector establece y a su vez, satisfacer las necesidades de la población en materia pedagógica.

Con relación a los cambios que se han llevado a cabo en materia de la enseñanza por proyecto en Venezuela surgió la necesidad de llevar cabo el presente estudio para dar respuesta al comportamiento que ha presen- 
tado la educación venezolana en los últimos años.

Es importante señalar que la finalidad de la investigación fue detectar los cambios que emergen en los docentes durante la implementación de un plan de formación pedagógica para la enseñanza de los proyectos desde la integralidad del ser humano.

MÉTODO

El estudio se desarrolló bajo el tipo de investigación interactiva con la modalidad investigación acción. El diseño se consideró de campo, transeccional, contemporáneo. La población de estudio estuvo conformada por 25 docentes entre guías y coordinadores de proyectos, según data institucional de la escuela en estudio.

Las técnicas de recolección de los datos utilizadas fueron la observación participativa mediante una guía de diagnostica donde se recolectó la información sobre los cambios que los docentes lograron luego de aplicado el programa de formación docente.

\section{RESULTADOS Y DISCUSIÓN}

Los resultados de este trabajo se presentaron en función de los logros obtenidos con la aplicación de un plan de formación pedagógica a los docentes, basado éste en los conocimientos que, desde la integralidad, debería tener un docente sobre los procesos pedagógicos. Los cambios se midieron a partir de acciones planificadas por el investigador las cuales se detallan a continuación:

Actividad 1: Reunión interactiva, entre investigadora coordinadores de proyectos, docentes guías y directivos.

Durante el proceso de desarrollo de la actividad a presentar, el personal asistente se mostró poco interesado, pese a la promoción que se realizó a la actividad; sin embargo, hubo participación de alrededor de un 50\% de los asistentes en la discusión que fue emergiendo durante el proceso. Además, se observó receptividad del personal directivo en cuanto al inicio del proceso de la formación pedagógica desde la integralidad del ser humano, lo que logró involucrar una gran parte del personal en la fase de sensibilización del proceso, tomando en cuenta que un grupo de docentes estuvieron dispuestos a iniciar la formación.

Actividad 2: Presentación y discusión de las 57 líneas orientadoras. 2014-2015 y 2015-2016.

La mayoría de los docentes y directivos presentes expresaron que ignoraban, en el primer período, la existencia de las normativas planteadas, por lo que, habían estado trabajando de acuerdo a criterios generados en la institución, y llegaron de esta manera, en muchas oportunidades, a crear confusión en lo que se debía realizar en el año escolar; por tal razón se observó durante el ejecútese de la primera actividad (charla), que el grupo asistente se mostraba interesado por saber qué deberían realizar, qué lineamientos tenían que seguir durante el proceso; lo que les permitió más claros en el ejecútese a seguir de los proyectos pedagógicos; sin embargo, luego del desarrollo de las diferentes actividades que se realizaron se evidenció aun cierta confusión entre los colegas, con respecto a cómo llevar a cabo cada uno de los lineamientos emanados por el ente rector en materia educativa.

\section{Observación recursiva}

La investigación permitió un ciclo de acción donde se analizó cada actividad realizada, de manera de poder evaluar las 
situaciones y luego se emitió una retroalimentación. En reuniones posteriores, se acordó con los docentes implementar un ciclo de conversatorios, con la finalidad de plantear los logros y debilidades que era necesario fortalecer durante la formación pedagógica.

Además de la situación de confusión que se presentó con la integración de los nuevos lineamientos donde se involucraron los proyectos pedagógicos, se anexó al contexto la emisión de normativas, en el mismo periodo de estudio y en el siguiente período, lo que llevó a la investigadora en conjunto con el equipo de acción a retomar otro ciclo de charlas para informar lo establecido $\mathrm{y}$ relacionarlo con los proyectos pedagógicos, específicamente en las escuelas técnicas.

No obstante, en el siguiente período de estudio, se emitieron otras líneas orientadoras, por lo tanto nuevamente surgió la necesidad de dar información al personal que estaba participando en las acciones; por tal razón se observó interés y disposición en la socialización de la misma y a su vez la conexión que tenían con los proyectos pedagógicos, ya que las pautas que se emitieron orientaron nuevamente las acciones a seguir en el desarrollo de la enseñanza de los proyectos en el periodo 2015-2016.

\section{Logros}

En función de que fue un algo complicado que los docentes que compartían el ciclo de acción con la investigadora colocaran en práctica lo plasmado por el ministerio de educación, se planificó un segundo encuentro de charla, en el cual se observó mayor fluidez y dinamismo debido a que las nuevas líneas orientadoras representaban para ellos innovaciones determinantes para el proceso, pero no cambios en lo ya interiorizado, esto permitió detectar en la investigación que, con las acciones entabladas para tal situación, había surgido el cambio que se buscaba en aquellos docentes que al inicio se mostraron resistentes y confusos; ya que se veían más abiertos y entendidos en lo que debían hacer.

Actividad 3: Taller de proyectos pedagógicos (PEIC, P.A y PSP) I y II Parte.

Durante el desarrollo del primer taller, se observó asistencia de la mayoría del personal docente, con inclusión de los docentes que no eran profesores guía para el periodo 2014-2015; se observó, de igual manera, un desconocimiento en lo que respecta a la estructura a seguir para el abordaje de los proyectos pedagógicos. Por lo tanto, en este segundo taller, a medida que se ejecutaban las ponencias, surgieron muchas inquietudes, lo que permitió una participación continua, que generó la formación de equipos de trabajo para estar en constante sintonía.

En función de la integración de los presentes se organizaron mesas de trabajo, donde cada docente se agrupó por área de conocimiento, iniciando la planificación de los diferentes proyectos pedagógicos que se implementaron durante el primer período del estudio. El ejecútese de esta actividad marcó el inicio de una enseñanza por proyecto desde la integralidad, ya que se había realizado un análisis de las planificaciones previas al año escolar cuando se inició la investigación y se evidenció una separación entre las áreas de conocimiento y los proyectos pedagógicos a realizar, dando paso a una nueva forma para planificar, en la que se tomaba en consideración lo planteado por la investigadora y el equipo de acción que se había conformado.

\section{Observación recursiva}

En vista de que el taller representó la guía que orientaría el trabajo de los docentes dentro de la institución estudiada, el mismo no llenó en su totalidad las expectativas del 
grupo, por lo tanto, durante los acompañamientos realizados, se registraron las debilidades que afloraron y en reuniones posteriores con los docentes se hizo mediación y se estableció compromiso para mejorar el trabajo dentro de los espacios de aprendizajes.

Seguidamente para el segundo período escolar 2015-2016, se realizó una segunda parte del taller de proyectos pedagógicos plegándose en función de las nuevas normativas emitidas durante ese momento, además de servir de reforzamiento en situaciones que se habían detectado durante el ciclo de observaciones. Ante lo cual el grupo específico de docentes guías y coordinadores de proyecto que permanecía en continua formación, estuvieron de acuerdo.

De allí, que los docentes en formación escucharon toda la información de los contenidos del taller a fin de realizar una nueva planificación que se pondría en práctica para el año escolar 2016-2017, pero con una actitud más clara de lo que debía realizar el docente tutor para la inserción de la integralidad del ser humano en la enseñanza de los proyectos planteados.

\section{Logros}

En función de lo planteado, se evidenció que el trabajo realizado durante los períodos en estudio, logró un cambio dentro de la práctica docente en la aplicación de los proyectos pedagógicos de la ETA Pedro José Hernández. Esto posicionó a la institución en los primeros lugares en la enseñanza por proyecto, entre las demás escuelas técnicas del entorno.

Actividad 3.1: Taller sistematización de experiencias de los proyectos pedagógicos. Nivel I y II.
El taller estuvo dirigido a docentes guías y coordinadores de proyectos, pero a pesar de que los docentes estaban encaminados en la formación de los proyectos debido al taller previo, se observó apatía en el personal. Sin embargo, al ejecutarse la acción mediante la sistematización de las experiencias, los docentes expusieron su asombro al creer que la sistematización solo se podía usar en los procesos de investigación y no en la educación.

\section{Observación recursiva}

Durante este proceso se mostró una situación débil en cuanto al desarrollo de la formación para los docentes que formaron parte del estudio; de acuerdo a ello, se plantearon alternativas para explicar individualmente a los docentes que expresaron no haber entendido como realizar el proceso de sistematización aplicado a los proyectos pedagógicos.

Por tal razón, a pesar de los esfuerzos implementados, para dejar claro por qué aplicar la sistematización y como realizarla, fue necesario iniciar un segundo proceso de sensibilización, para dar a entender al colectivo, la importancia del proceso de sistematización en el campo educativo, especialmente para la enseñanza basada en proyectos.

Sin embargo, aún se mantenía un número significativo de docentes que tenían poco conocimiento del proceso de sistematización de las experiencias; ya que se confundían con el registro de información. Se procedió a realizar el taller, pese a la incomodidad del grupo, pero a medida que se desarrollaron los contenidos, surgió una discusión positiva que llevó al logro del objetivo planteado, esta situación permitió que los docentes realizaran producciones en el taller; donde los presentes sistematizaron como ejemplo práctico, experiencias propias 
vividas en el aula de clase y dentro de su vida cotidiana.

\section{Logros}

Los docentes guías que compartieron el proceso de investigación en conjunto con los coordinadores, que para ese momento fueron pieza fundamental en el desarrollo de la formación, expresaron su comprensión en lo que respecta a cómo aplicar dentro de la enseñanza por proyecto el proceso de sistematización de experiencia, tal y como lo exige el ministerio del poder popular para la educación dentro de las líneas orientadoras.

Actividad 4: Taller de competencias docentes y los proyectos pedagógicos.

El taller estuvo dirigido a todos los docentes guías y coordinadores de proyectos que conformaban el colectivo de la institución para los periodos escolares en curso. En el transcurso del taller se observó, que el docente confundía las competencias con los objetivos; de allí, que el ponente tuvo que aclarar primeramente dichos términos.

La acción a ejecutar, estuvo dirigida a orientar sobre las competencias pedagógicas que deben poseer los docentes para el ejecútese de su labor en el aula, específicamente durante el trabajo con los proyectos, además indagar las competencias que los docentes formulaban en la clase.

Por lo tanto, previo diagnóstico, la investigadora observó que los docentes tienden a identificar, describir y analizar dentro de las actividades de las clases a realizar, pero las planificaciones previas analizadas, muestran que durante el ejecútese pasan por alto competencias pedagógicas relacionadas con el hacer y el conocer.

No obstante, durante el taller los presentes mostraron iniciativa por realizar preguntas; de cómo podían ellos desarrollar competencias y a su vez relacionarlas con los contenidos que debe saber el docente para abordar la enseñanza de los proyectos, tomando en cuenta la integralidad del ser humano.

\section{Observación recursiva}

Durante el desarrollo del taller como acción de formación se detectó, que el docente necesitaba comprender lo que es una competencia y además identificar la que ellos mismos han desarrollado o practican, de manera tal, de poder fortalecerlas y aplicarlas en la enseñanza de los proyectos pedagógicos, para tal situación se planificó con los coordinadores de proyecto una segunda acción, en función de seguir en el estudio de la temática y poder contribuir al cambio.

Actividad 4.1: Seminario sobre competencias docentes y los proyectos pedagógicos.

Durante el desarrollo del seminario, se conformaron grupos que tenían como función analizar los aspectos que se relacionaban con las competencias del docente y los proyectos pedagógicos. Durante el proceso se observó que los docentes se mostraron sorprendidos ante las debilidades que presentaban con respecto a las competencias docentes necesarias en su praxis, más aún en la enseñanza de los proyectos pedagógicos. Los docentes asistentes discutieron y presentaron posibles alternativas para mejorar la situación y asumieron el compromiso de tomar en cuenta lo planteado.

Las coordinaciones en conjunto con los docentes acordaron atender todas las dudas que emergían durante el ejecútese de los proyectos pedagógicos.

\section{Logros}

Durante el seminario, emergieron explicaciones por parte del colectivo docente, 
que evidenciaron que utilizaban en su práctica docente competencias del ser, lo que indicó una fortaleza para la enseñanza de los proyectos desde la integralidad de la persona.

Actividad 5: Disertación interactiva, sobre los aspectos básicos en el contexto integral del estudiante como ser humano.

Durante la presente acción, se difundieron, las dimensiones que integran el todo del ser humano. En el desarrollo de la actividad se observó una atención continua de la mayoría de los asistentes al foro; lo que llevó, a un desarrollo interactivo, donde no solo se explicó la temática pautada, sino que los presentes realizaron diversas preguntas, además indicaron haber aclarado parte de sus inquietudes.

Algunos de los asistentes, mostraron estar muy pasivos ante la explicación, pero otros docentes refirieron que desconocían las dimensiones del ser humano y su integración con la enseñanza de los proyectos, ante lo cual se consiguió que la acción permitiera mejorar la concepción de la integralidad en la práctica pedagógica.

Actividad 6: Acompañamiento en el desarrollo de la enseñanza de los proyectos.

Luego de haber realizado, la formación pedagógica con los docentes del colectivo de proyectos y que se tomó en cuenta lo explicado durante el proceso, así como lo planificado, se llevó a cabo una serie de acompañamientos pedagógicos en los espacios de aprendizajes, para constatar que los docentes incluyeran en sus prácticas de enseñanza los conocimientos adquiridos durante la formación.

Además, durante la visita a las clases de los docentes que se integraron a la formación, se observó la puesta en práctica de algunas competencias relacionadas al hacer y al conocer; aunado a eso, se observó que el docente trató de integrar en su praxis pedagógica las dimensiones del ser, con la inclusión de la dimensión biológica y la volitiva, la cual generalmente se obviaba durante el proceso de enseñanza $y$ aprendizaje.

De igual manera, al revisar el cuaderno de registro de clase del docente se observó que incluyó el proceso de sistematización de las experiencias en el desarrollo de las clases de los proyectos.

En cuanto a las estrategias señaladas por los docentes, éstas estaban más acordes al trabajo por proyecto, debido a que los docentes presentaban menos confusiones con respecto a las estrategias y técnicas didácticas, cuestión que al inicio de la investigación era muy evidente entre los docentes que estuvieron en la formación.

Entre las estrategias más usadas se observó, la comunicación de contenidos y el fomento del trabajo colaborativo; además se evidenció la puesta en práctica de técnicas didácticas como: el empleo de organizadores de clasificación, integración de los contenidos previos, el uso de analogías y la elaboración de cuadros sinópticos, además se observó en la mayoría de los docentes visitados, que promovían las discusiones guiadas. También se evidenció con frecuencia, la explicación verbal, el fomento de la pregunta y la respuesta, además de agrupar a los estudiantes.

\section{Actividad 7: Encuentro de saberes}

Durante estas actividades, de la cual algunas fueron dentro del plantel y otras fuera de la institución, docentes y estudiantes mostraron los trabajos realizados en su proceso de enseñanza de los proyectos desde la integralidad del ser humano, lo cual fue un indicador importante de los cambios emer- 
gentes, luego del desarrollo de la formación pedagógica que se llevó a cabo en el contexto de estudio.

Se observó de esta manera, en los eventos de intercambio entre los docentes de las instituciones técnicas y liceos integrales que forman parte de los diferentes rincones del municipio y del estado, la participación entusiasta de la mayoría de los docentes guía y la presencia de los coordinadores de proyectos de las instituciones, quienes mostraban con dedicación y entusiasmo el producto de su praxis pedagógica llevada a cabo dentro de sus espacios de aprendizaje.

\section{Ciclo de Acción-reflexión-acción}

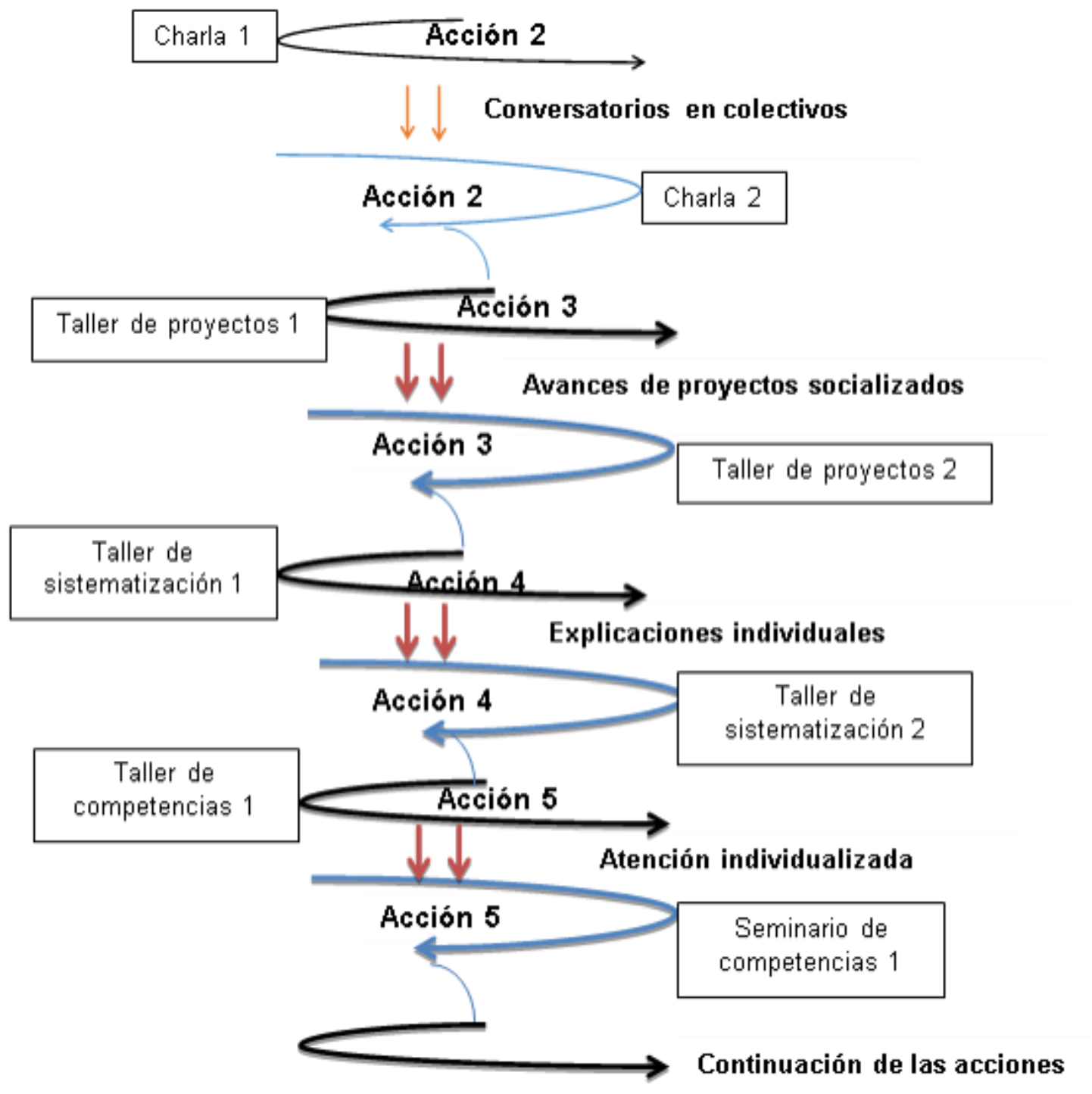

Figura 1: Acciones aplicadas para abordar situaciones débiles durante las acciones 


\section{Discusión}

\section{Análisis de las concepciones en la enseñanza por proyectos}

Uno de los cambios del sistema educativo venezolano fue la implementación del Currículo Bolivariano, donde se plantea la perspectiva de un hombre, humanístico, trabajador, transformador y forjador de su propio futuro, lo cual responsabiliza a la escuela de la formación de un ser humano integral y responsable; de acuerdo a ello, las instituciones del país dentro de sus diferentes sistemas y modalidades permanecen desde el año 2007, bajo los criterios de la educación bolivariana, con una filosofía de educación dialógica $\mathrm{y}$ transformadora. Este nuevo sistema según el Currículo Bolivariano, (2007):

constituye un elemento primordial para la construcción del modelo de la nueva República; en la medida en que está compuesto por un conjunto orgánico de planes, políticas, programas y proyectos estructurados e integrados entre sí, que orientado de acuerdo con las etapas del desarrollo humano, además persigue garantizar el carácter social de la educación a toda la población venezolana desde la rectoría del Estado venezolano, ejercida por intermedio del Ministerio del Poder Popular para la Educación (p.21)

En este sentido, el actual Currículo Bolivariano está conformado por los subsistemas: educación inicial bolivariana (niveles maternal y preescolar); educación primaria bolivariana (de $1^{\text {o }}$ a $6^{\underline{0}}$ grado); educación secundaria bolivariana, en sus dos alternativas de estudio (liceo bolivariano, de $1^{\circ}$ a $5^{\circ}$ año; y escuela técnica Robinsoniana Zamorana, de $1^{\circ}$ a $6^{\circ}$ año); educación especial; educación intercultural y educación de jóvenes, adultos y adultas; siendo entre los diferentes niveles y modalidades, la educación técnica eje primordial para el cumplimiento de los objetivos planteados en el currículo.

Por su parte, el nivel de escuelas técnicas presenta cambios productivos en su praxis pedagógica guiado por la filosofía de Simón Rodríguez, "inventamos o Erramos", transformándose dentro del enfoque pedagógico de aprender haciendo, lo que origina en estas instituciones, ahora denominadas, escuelas técnicas Robinsonianas, la necesidad de reorientar al educando dentro de una formación basada en proyectos como propuesta integradora de los contenidos en busca de la formación de un ser integral.

De acuerdo a esto, la formación dentro de la primicia de los aprendizajes basados en proyectos, debe ser el común de las escuelas que pertenecen al subsistema medio técnico, ya que como lo plantea el currículo actual venezolano (2007).

La escuela técnica Robinsoniana y
Zamorana tiene como finalidad
ofrecer una formación dirigida al
desarrollo endógeno, fomentar
habilidades, destrezas, valores y
virtudes que fortalezcan esta
orientación; al mismo tiempo
propiciar el pensamiento crítico,
reflexivo, humanista, liberador y
ambientalista, características espe-
cíficas que forman partes de la
praxis educativa nombrada de
acuerdo al sistema social actual.
(p.28)

En función de esto, las escuelas técnicas en el marco del currículo bolivariano (2007), forma a los estudiantes, durante seis (6) años, otorgándoles al finalizar, el título de técnico medio; para ello, el área de formación especializada en esta alternativa de estudio, define el campo ocupacional; por lo tanto, la 
formación de los y las estudiantes en las escuelas técnicas, responde al plan de desarrollo nacional, en la medida en que contribuye al proceso productivo, la producción de bienes y servicios, la innovación y la independencia tecnológica.

Ahora bien, en estos entornos educativos, dentro de los lineamientos actuales para el nivel profesional, como lo plantea el Manual de Escuelas Técnicas (2007), se concibe la educación y trabajo como procesos fundamentales para alcanzar la defensa y desarrollo de las personas, el respeto a su dignidad y el ejercicio democrático; por tal razón, estas instituciones están inmersas dentro de la nombrada praxis de la enseñanza $\mathrm{y}$ el aprendizaje basado en proyectos en función de formar un estudiante orientado por la filosofía del "aprender haciendo" ya que, día a día tienen bajo su responsabilidad la preparación de la mano de obra de los hombres que formaran parte de la población productiva del futuro.

El proceso de aprendizaje basado en proyectos, está orientado por la idea, de que el aprendizaje se obtiene mejor, cuando es el resultado de experiencias significativas para el estudiante, dado que este se hace participe en su planeación, creación y entendimiento, específicamente a través de los proyectos orientados por el profesor, con base en la hipótesis de que los intereses del estudiante deben ser el cimiento para realizar los proyectos y estos a su vez el instrumento central en el proceso de Enseñanza $y$ aprendizaje.

En esta práctica el estudiante es el actor principal de su aprendizaje, lo cual implica que debe desarrollar un proceso de autoaprendizaje, por cuanto se le da igual importancia a los conocimientos de formación de aptitudes y actitudes, pero a su vez el docente es consecuentemente el equilibrio del proceso, porque es quien sin olvidar que el estudiante es un ser humano único, facilita a través de la enseñanza los objetivos pautados para la ejecución de los mismos.

Esta praxis del docente reconoce la integralidad del ser humano; ya que como lo explica Barrera (2013) en la educación se debe propiciar una reflexión que sustentada sobre los principios de la unidad, unicidad y universalidad se plantee la interpretación de lo antropológico con criterio integrativo, la cual dicho se concreta en la comprensión integral del hombre; es decir los procesos planificados deben estar direccionados de acuerdo a las dimensiones que hacen integral a un individuo.

Por tal razón, las prácticas pedagógicas además de estar asociadas a las normativas que rigen el ente gubernamental educativo, debe adaptarse a las etapas que corresponden a cada individuo dentro de sus dimensiones, así como a sus limitaciones, presentes en sus entornos sociales con la mera finalidad de formar un hombre apto para el desenvolvimiento social, que pueda solventar las vicisitudes que se generen en sus ambientes.

En relación a ello, el Ministerio para el Poder Popular de la Educación en Venezuela, ha activado formaciones para todo el personal de la enseñanza de los proyectos, como respuesta a las diferentes dudas que los docentes han expuesto tener sobre esta nueva forma de trabajo.

El proceso de enseñanza y aprendizaje por proyectos es un binomio en equilibrio, sin embargo, es en la enseñanza donde se hace necesario enfocar la atención, para obtener resultados positivos, ya que el docente es quien dicta los criterios durante el recorrido pedagógico para que los estudiantes alcancen el aprendizaje.

Consecuentemente, es menester señalar que el docente debe estar preparado para afrontar diferentes retos en la profesión, por 
lo que es indispensable alimentar la inquietud de construir su aprendizaje, lo que amerita una formación pedagógica constante, como lo refiere Amilburu y Gutiérrez (2012):

La formación de los educadores es una tarea que no puede darse nunca por concluida, por ello, una de las principales preocupaciones de estos profesionales debería ser su propia mejora, ya que uno de los peligros que asecha a los educadores, es sentirse totalmente preparado para realizar sus tareas, conformándose con los conoci-mientos y destrezas que posee sin tomar en cuenta que quien no se esfuerza por avanzar, retrocede. (p. 191).

Debido a esto, el profesional de la docencia debe estar claro que la formación pedagógica no solo implica la culminación de la carrera docente ni la matriculación en cursos, sino que es el continuo crecimiento desde sus espacios de aprendizajes, ya que la tarea de enseñar realmente consiste en facilitar el aprendizaje del educando, lo cual convierte la enseñanza en un proceso complejo que requiere de competencias específicas de acuerdo a los grupos enrumbados.

Sin embargo, a pesar de la constante promoción y formación de la planteada praxis pedagógica, en la institución objeto de estudio de esta investigación, la Escuela Técnica Robinsoniana de Salud Pedro J. Hernández, se observó que los docentes presentaban dificultades para abordar este proceso de enseñanza, ya que persistían en la utilización de prácticas pedagógicas no acordes con la enseñanza de proyectos. Aunado a ello, las normativas emanadas del ente rector, no siempre estaban en consonancia con la praxis que ejecutaba el docente en las aulas de las instituciones, especialmente en las escuelas técnicas, ya que el proceso de enseñanza por proyectos se desarrollaba en forma paralela a la aplicación de los contenidos curriculares individuales, lo cual ocasionaba que en el proceso de interacción docente - estudiante se obviaran las dimensiones del ser, por lo que no se consideraba al estudiante como una persona integral.

En este sentido, surgió la inquietud de abordar este estudio y se planteó la siguiente pregunta de investigación ¿Qué cambios generará un plan de formación pedagógico en los docentes de la enseñanza de los proyectos, de la escuela técnica de promoción social y servicio de salud, Pedro José Hernández, ubicada en la parroquia Ambrosio del municipio Cabimas estado Zulia?

El ser humano desde el inicio de su historia, siempre ha buscado la comprensión y transformación de su entorno; para ello ha ideado uno de los procesos más importantes para el desarrollo y socialización del hombre, la educación. Según lo planteado, la educación implica la innovación, para que la sociedad evolucione, por lo tanto, no debe simplemente adaptar al hombre a su contexto de vida; sino, amerita un proceso donde se enseñe al hombre a ser crítico y reflexivo desde la pluralidad de las filosofías.

No obstante, el proceso de la educación no es más que una acción de necesidad continua del hombre, que permite su humanización social; lo cual indica que debe orientarse hacia las posturas filosóficas que tenga el entorno, para un momento determinado y además guiar al educando por los principios pedagógicos, que permiten de una manera equilibrada el proceso de enseñanza y aprendizaje, dentro de los espacios educativos.

En este sentido, el Sistema Educativo venezolano se ha mantenido en pro del desarrollo de la nación desde el decreto de la educación formal gratuita, lo cual permite su diversificación por todo el territorio nacional con la intensión de que todo venezolano 
pueda acceder a ella; además de esto, los entes gubernamentales en sus diferentes formas de gobierno han establecido cambios curriculares en función de mejorar el proceso de enseñanza y aprendizaje, lo que facilita la praxis del profesional de la educación y el desenvolvimiento de los educandos.

Al respecto, las acciones planteadas y puestas en marcha se orientan a dar cumplimiento a lo estipulado en el artículo 103 de la Constitución Nacional (1999) donde se específica que:

Toda persona tiene derecho a una educación integral de calidad, permanente, en igualdad de condiciones y oportunidades, sin más limitaciones que las derivadas de sus aptitudes, vocación y aspiraciones. La educación es obligatoria en todos sus niveles, desde el maternal hasta el nivel medio diversificado. La impartida en las instituciones del estado es gratuita hasta el pregrado universitario. (p.21)

Es así como desde los principios educativos dados por la constitución nacional se plantean numerosos esfuerzos por mejorar el sistema educativo; debido a esto, existen una diversidad de variados programas, planes, proyectos y otras estrategias, para cumplir con los diferentes objetivos del sistema formal. En concordancia con esto, la Ley de educación (2009) en su artículo 6, hace referencia al derecho pleno de la población de tener una educación integral, permanente, continua y de calidad para todos y todas con equidad de género en igualdad de condiciones y oportunidades, derechos y deberes.

De esta manera, se plantean diferentes posiciones en cuanto a cómo debe ser el proceso educativo actual del país, en busca de la perspectiva de un ciudadano para una sociedad humanista e integradora. En confor- mación con lo planteado, el maestro, está llamado a optimar su praxis con el propósito de que se transforme en aprendiz constante, lo que origina que el personal de la docencia sea un investigador pedagógico con proceso de actualización constante y voluntario.

Dentro de ese marco de ideas es necesario mencionar que Venezuela es merecedora del Currículo Nacional Bolivariano (2007), el cual plantea en sus objetivos, la generación de un proceso educativo que permita caracterizar, reflexionar y analizar la realidad para transformarla, además de ello, induce a promover la independencia cognitiva, apropiación de los saberes; permitiendo un pensamiento autocrítico, crítico y reflexivo; así como el interés por la ciencia, la tecnología, el conocimiento y la innovación; y su aplicación, desde una perspectiva social que favorezca el trabajo liberador, como herramienta para el desarrollo económico, social y político del país, en busca de la seguridad y soberanía nacional.

De acuerdo a lo expresado, el Currículo Nacional Bolivariano postula una estructura pedagógica basada en la formación de aprendizajes por proyectos, los cuales nacen a principio del siglo $\mathrm{XX}$ en un movimiento progresista estadounidense donde se organiza el método de proyectos, basado en la teoría de Dewey que nace a finales del siglo XIX como el primer modelo pedagógico que se sustenta en la experiencia empírica.

En función de esta primicia el aprendizaje basado en proyectos ha sido difundido en América latina especialmente a movimientos de educación popular, en busca de una educación, basada en los enfoques de la teoría constructivista, que oriente al ciudadano del nuevo siglo hacia la dimensión del "ser integral" capaz de orientar su propio 
aprendizaje y llegar a ser protagonista de su propia transformación social.

En razón de lo mencionado, el Currículo Bolivariano (2007), plantea como proyectos, a una forma de organización del aprendizaje en la que maestros, maestras, estudiantes y familia buscan, en conjunto, solución a un problema de su interés, preferiblemente con relevancia social, mediante un proceso activo y participativo.

De esta manera, los proyectos pedagógicos, que sustenta el actual currículo venezolano, son una actividad dentro del plan de estudio que de manera planificada ejercita al estudiante en la solución de problemas cotidianos, seleccionados por tener relación directa con su entorno social, cultural científico y tecnológico. En este sentido Novoa (2005), expresa que a través de este tipo de práctica educativa, el docente y el estudiante trabajan en equipo de manera que pueden ayudarse en la construcción de un conocimiento desde su propia realidad.

Basado en esta perspectiva, el aprendizaje por proyectos es visto como una estrategia que induce al docente y al estudiante al trabajo liberador, así como a la búsqueda de la comprensión de su entorno. De esta manera, los estudiantes orientados bajo las concepciones pedagógicas del actual Currículo Bolivariano, dirimen su aprendizaje a través de proyectos los cuales sin ser una práctica nueva posee características innovadoras que consideran al estudiante como uno de los actores principales del proceso, sin perder de vista la importancia del papel que juega el docente como responsable de un proceso de enseñanza innovador y productivo, por ser él quien facilita las pautas para el ejecútese adecuado del aprendizaje

El aprendizaje por proyecto se sustenta en la teoría constructivista, en la que según Amilburu, (2011), se concibe al aprendizaje como un proceso en el cual el estudiante elabora activamente los nuevos contenidos, mediante el apoyo de sus conocimientos previos, es decir el aprendizaje se produce al edificar el saber desde su propia experiencia, ya que aprender es un proceso constructivo.

Por su parte, Soler (2006) expresa, que "educar es aprender experiencias significativas y relevantes para el individuo" (p.42), o lo que es lo mismo durante el proceso de educación los individuos construyen su propio conocimiento a través de la obtención de experiencia debido a la constante actividad que desarrollan día a día.

Es así como esta visión de la educación, se alinea con la nueva concepción del aprendizaje por proyectos, al ser éstos considerados como una oportunidad que induce al docente y al estudiante a transitar el camino de la enseñanza aunado a los contenidos y significados de experiencias compartidas desde la integralidad, todo desde el hacer, pero con inclusión de la conciencia del ser, lo cual implica privilegiar el diálogo, el compromiso, la reflexión, la pregunta pero también la respuesta.

Desde esta nueva concepción curricular, las escuelas venezolanas llevan a cabo la enseñanza por proyecto en sus diferentes tipos como son: Proyecto educativo integral comunitario (PEIC), Proyectos de aprendizajes (PA), Proyecto de desarrollo endógeno y Proyecto pedagógico productivo.

En este sentido, Gonzales y Escribano (2004), establece que el término enseñanza proviene etimológicamente de insignare, que significa dar signo a alguna cosa o realidad, mostrar o exponer una cosa para que sea vista y apreciada. Indudablemente según el autor, hay enseñanza que son didácticas y otras que no lo son; o lo que es lo mismo, no reúnen las características cualitativas exigibles para que se le pueda denominar como enseñanza y específicamente como enseñanza educativa. Este otro tipo de 
enseñanza son las relativas a reconocer al estudiante como una persona, que tiene dimensiones tales como: intelectiva, ética y biofisiológica.

Novoa (2005), hace referencia a la dimensión pedagógica relacionada con los métodos, recursos de enseñanza y aprendizaje utilizados por los docentes para la enseñanza. Es en esta dimensión en la que se aprende a enseñar y aprender, por lo tanto, es sin duda el escenario para que el docente tutor desarrolle métodos pedagógicos de apoyo al aprendizaje, propios de él y de los estudiantes en el contexto de la práctica educativa y en el marco de la producción. En esta dimensión los docentes son actores en el aprendizaje de nuevos métodos y prácticas pedagógicas pertinentes al medio con distintos tipos de interlocutores y en el contexto del desarrollo.

Para tal efecto, la enseñanza, es un proceso de sumo interés y cuidado en el desarrollo de la profesión docente, porque para que haya educación, debe haber aprendizaje y este en forma general depende de la manera que el docente elija para enseñar. De esta manera la enseñanza por proyecto es una manera de orientar el aprendizaje con los estudiantes dentro de los espacios educativos y amerita una formación docente acorde con los requerimientos del proceso.

Para Novoa (2005), la enseñanza implica saber lo que se va a enseñar, que argumentos presentar, que respuestas aguardar y en qué momento se debe recalcar algo. Ante lo cual la formación pedagógica constituye una especie de empoderamiento de elementos didácticos básicos e indispensables en el quehacer docente a fin de estar seguros en los pasos a lograr para abordar la enseñanza. De allí, que la selección del método es uno de los primeros pasos de la acción educativa ya que éste hace más fácil la transmisión de los contenidos del aprendizaje. Según López (2005) "los métodos didácticos, son el conjunto de procedimiento empleados para desarrollar las actividades formativas" (p. 204).

En efecto, la selección de los elementos didácticos es un punto crucial $\mathrm{y}$ de gran envergadura en el proceso educativo, ya que éstos son las herramientas con que cuenta el maestro y el estudiante; herramientas que no son inventadas, sino que se generan del contacto continuo, del dinamismo humano, y a su vez se fortalecen y actualizan en el alcance de los objetivos planteados.

Según las posiciones de los autores citados, la enseñanza por proyecto es una estrategia formativa que orienta el aprendizaje de los estudiantes en las instituciones educativas, donde se plantea como un proceso didáctico que se lleva a cabo mediante un conjunto de procedimientos dirigidos por profesionales con sólidos conocimientos peda-gógicos.

Al respecto Muñoz (2012), estipula que el proceso de enseñanza requiere reflexión y análisis constante y se debe pensar sobre la práctica, antes, durante y después de la misma. Es decir, este proceso realmente no debe ser motivo de improvisación, es necesaria la reflexión de cada paso a seguir, aunque también es menester señalar que es necesaria una planificación con un margen de flexibilidad.

En función de esto, la planificación servirá para expresar de forma gráfica o escrita, el orden en que deberán ser desarrolladas unas series de actuaciones en pro de lograr con éxito el fin de un proyecto. Es decir que puede verse como la orga-nización que incluye la programación de todo lo planteado para evitar perderse y desviar los objetivos que previamente fueron organizados. 
Asimismo, Núñez y otros (2014), plantean que planificar la enseñanza, significa tomar consideración de las determinaciones legales, los contenidos básicos de la disciplina, el marco curricular en que se ubica la disciplina, la visión propia del docente y las características de los estudiantes (tales como el número, su preparación anterior y sus intereses) y finalmente los recursos disponibles. Así la planificación, consiste en analizar las diversas tareas que configuran, la totalidad de un proyecto y a su vez establecer anticipadamente las relaciones lógicas que deben regir entre ellas.

En este sentido, el primer paso de un docente antes de iniciar un proyecto es el diseño de la instrucción, la cual le guiará por el recorrido para lograr lo planificado. Al respecto, García (2013), expresa que para lograr que todas las fases del proyecto se desarrollen con normalidad se requiere de una cuidadosa planificación que concrete los procesos curriculares en el día a día de las aulas. No obstante, los elementos de un proyecto, no son diferentes de los de una unidad didáctica, o un centro de interés, sin embargo, lo que, si requiere un tratamiento especial, es la participación del estudiantado en la toma de decisiones en cada uno de sus componentes.

En el trabajo por proyecto, es común que se vincule, como una estrategia de enseñanza y aprendizaje, el desarrollo práctico del mismo con un trabajo basado en la indagación; por ello, para llevarse a cabo el ejecútese del proceso de enseñanza por proyecto según Tobón (2006), los estudiantes, con apoyo y mediación del docente, ejecutan el proyecto diseñado en la fase anterior, buscando el logro de las metas acordadas tanto en lo referente al desarrollo de las competencias como la resolución del problema.
Resulta claro que para ello es vital en los espacios de aprendizaje, una organización minuciosa por parte del docente, tomando en cuenta el aspecto integral del estudiantado, debido a que una enseñanza de esta índole, según lo expresa Barrera (2013), indica una educación holística, debido a "que se presenta como una oportunidad para la realización integral, en donde la persona participa de la tarea de formarse a sí misma todo el tiempo y en todas las edades" (p.82).

Desde esta perspectiva, la integralidad se refiere a la compresión holística de la educación, la cual implica tener en cuenta al humano como lo que es; es decir el principio, centro y fin de la actividad formativa. En este sentido, todo proceso de enseñanza debe estar acompañado de estrategias, técnicas y tácticas; o lo que es lo mismo, elementos pedagógicos necesarios para el proceso los cuales deben estar acorde a las dimensiones del individuo a formar.

Igualmente, la autora explica que, a través de la holística, existe, el sentido de la libertad y la autonomía de cada persona; es decir, este propósito se concreta en la comprensión integral del ser humano la cual sostiene que la persona manifiesta su multidimensionalidad; que realmente se expresa como una condición integrativa donde están presentes variadas dimensiones o expresiones.

Al hablar de dimensiones, hace referencia a:

a) Dimensión biológica: es decir la corporeidad, la materialidad, los órganos los sentidos internos y externos, los sentimientos, los afectos, las emociones.

b) Dimensión Intelectiva: Expresada a través del pensamiento, del raciocinio, de la idea, de la abstracción, de la simbolización.

c) Dimensión Volitiva: Expresa a través de la capacidad de decisión de las acciones y 
de la sociabilidad, aquí también se expresa la condición ética y la valoración de las acciones y de lo que la persona es y hace.

\section{CONCLUSIONES}

El docente de las escuelas técnicas pertenecientes al contexto de estudio, no gozaban de un programa de formación sustentado en ciclos de formación pedagógica que orientara su práctica educativa basada en el trabajo por proyecto.

Los docentes de la institución iniciaron su trabajo por proyecto con una formación pedagógica orientada a la enseñanza por asignatura.

La falta de formación pedagógica del docente interfirió la enseñanza por proyecto lo cual impide que éstos se desarrollaran bajo la perspectiva del desarrollo integral de la persona.

El proceso de formación que se desarrolló en esta investigación correspondió al inicio de una espiral de recorrido para que el docente comprendiera e internalizara la importancia de sus competencias para el desarrollo de los proyectos.

\section{REFERENCIAS}

Asamblea Nacional Constituyente (1999) Constitución de la República Bolivariana de Venezuela. Distribuidora Escolar, Caracas.

Amilburu, M. G., y Gutiérrez, J. G. (2012). Filosofía de la educación: Cuestiones de hoy y de siempre (Vol. 4). Narcea Ediciones.

Amilburu, M. G. (2011). Teoría y práctica del análisis pedagógico del cine. Editorial UNED.
Asamblea Nacional (2009). Ley orgánica de Educación. Caracas.

Barrera, M (2013) Hologogía. Tercera Edición. Ediciones Quirón. Caracas

De Educación, L. O. (2009). Gaceta oficial de la República Bolivariana de Venezuela, 5.929. Extraordinario). Agosto, 15, 2009.

Felfeto, A (2014) La educación en el siglo XXI. Ediciones LuluPress. España

García-Ruiz, R. (2013). Enseñar y aprender en Educación Infantil a través de proyectos (Vol. 17). Ed. Universidad de Cantabria.

Gonzales, A., y Escribano, A. (2004). Aprender a enseñar: fundamentos de didáctica general. Editorial gráficas Cuenca SA, universidad de Castilla. La Mancha, 2.

Ministerio del Poder Popular para la Educación (2007). Currículo Nacional Bolivariano. Venezuela

Ministerio del Poder Popular para la Educación (2007). Manual de Escuelas Técnicas Robinsonianas. Venezuela

Muñoz, A (2012) Caos o planificación. Estrategias para el docente. Volumen 7. Buenos Aires

Novoa A. (2005). Formulación de proyectos productivos para el aprendizaje. Modulo (I) para el aprendizaje. IICA. Colombia.

Núñez, N., Vigo, O., Palacios, P., y Arnao, M. (2014). Formación universitaria basada en competencias: Currículo, Estrategias Didácticas y Evaluación. Chiclayo-Perú: Ediciones Usat.

López J. (2005) Planificar la formación con calidad. Ediciones epise. España

Peiró, S (2005) Nuevos desafíos de la Educación. Tomo II

Soler, E. (2006) Constructivismo, Innovaciones y Enseñanza efectiva. Editorial Equinoccio. Venezuela.

Tobón, S. (2006) Formación Basada en Competencias. Segunda Edición. EGOE: Colombia 